\author{
Military Technical College \\ Kobry El-Kobbah, \\ Cairo, Egypt
}

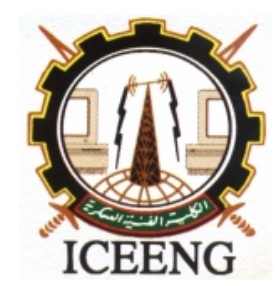

\section{$7^{\text {th }}$ International Conference on Electrical Engineering ICEENG 2010}

\title{
Phase gradient Autofocusing Technique (PGA)
}

\author{
By
}

\author{
A. S. Amein, F. M. Ahmed, M. Adel Ahmed, N. G. Mikhail *
}

\begin{abstract}
:
The phase-gradient autofocusing algorithm (PGA) has been widely used in spotlight Synthetic Aperture Radar (SAR) to compensate motion-induced blurs in the images. The PGA algorithm has proven to be a superior method for higher order autofocusing because it does not assume a model for the phase error. In this paper, a proposed pc-based software model of the PGA is introduced. Simulation results between blurred and focused images, or between the input phase error function and the estimated one carried out by calculating the rms value of the residual phase error, show significant removal of highorder phase errors and better removal of the low-order phase errors. Finally, a suggested graphical user interface (GUI) of the proposed algorithm for easier handling on pc-based equipments is introduced.
\end{abstract}

\section{Keywords:}

SAR, PGA, MoCo, GUI.

\section{Introduction:}

The Synthetic aperture radar (SAR) has the capability of producing high-resolution images in all weather conditions. It is rapidly becoming a key technology in modern remote-sensing applications. SAR is a coherent imaging system, which means that it is necessary to maintain the correct and precise phase relationship between return signals in order to perform coherent summation [1]. This requires precise motion compensation because uncompensated motion between the SAR antenna phase center and the scene being imaged causes phase errors that may blur the image severely. Even in a SAR equipped with modern electronic navigation systems, determining the platform position to the required tolerance over the entire synthetic aperture can prove to be a difficult task. This is especially true for the high-resolution imaging system since a long synthetic aperture is required. So it is necessary to adopt a data-driven autofocus technique to eliminate these phase errors. One technique that has been used to focus spotlight images is The PGA algorithm [2].

* Egyptian Armed Forces 


\section{PGA Fundamentals:}

An autofocus algorithm tries to estimate the unknown phase error function (PEF) by using the radar data themselves. The PGA algorithm estimates the phase error for each azimuth position along the synthetic aperture [3]. This technique is flexible in the sense that there is no limit to the order of the phase correction function. In principle, an arbitrary phase error curve can be estimated. In the literature it is claimed that this technique is quite robust and should give good results even with modest image contrast. Its basic premise is that all image points share the same underlying phase error, and that averaging the (residual) phase histories of many selected points will reveal the common error. PGA is an iterative technique that estimates the phase error from the data, applies this error to the data (corrects the phase) and again calculates the remaining phase error [4]. When the subsequent errors become smaller than a certain limit, the loop is ended and the PEF selected.

\section{PGA algorithm processing steps:}

The PGA algorithm is performed through four crucial processing steps to allow robust phase error estimation and image restoration. These four processing steps are referred to as center (circular) shifting, windowing, phase gradient estimation, and iterative correction. A block diagram for the PGA is shown in Fig.1.

The input data to the algorithm is the complex phase degraded SAR image. This implies that it is not necessary to know how the SAR image was formed, only that the image be phase coherent over the entire scene to be processed, this means that the complex image and phase history are Fourier transform pairs [5].

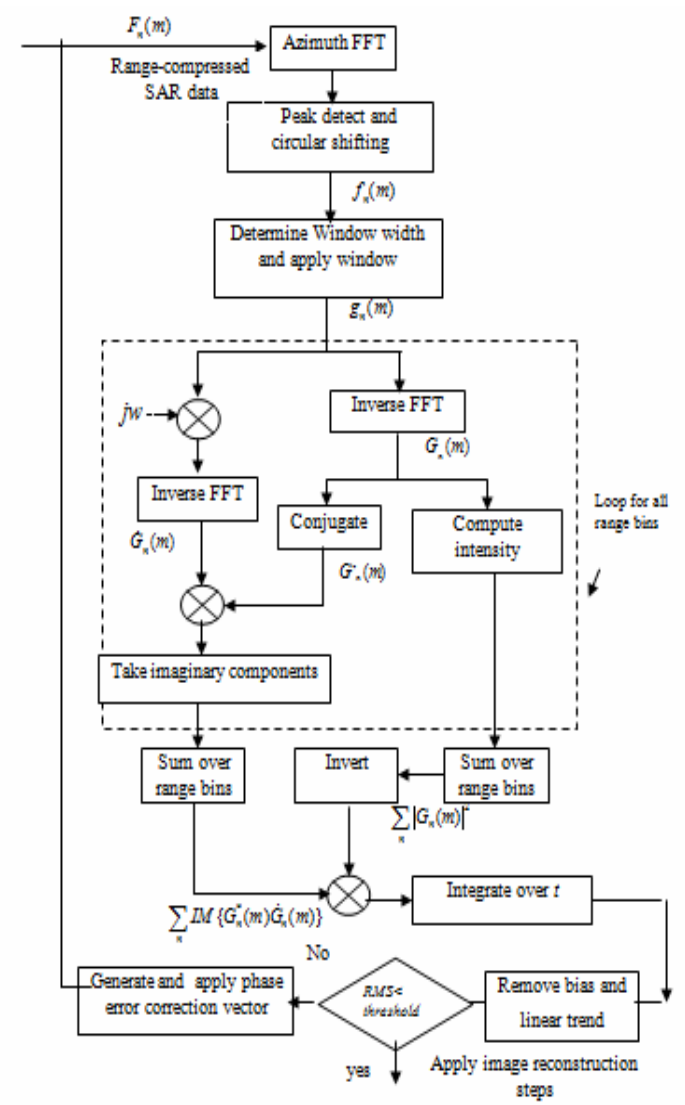

Fig. (1) Block diagram of the Phase Gradient Autofocus Algorithm 
A range-compressed blurred SAR signal at the $n^{\text {th }}$ range bin can be written as

$$
F_{n}(m)=\left|F_{n}(m)\right| e^{j(\phi n(m)+\phi e(m))}
$$

Here $\mathrm{n}$ is the range bin index, $\mathrm{m}$ is the Azimuth line, $\left|F_{n}(m)\right|$ and $\phi_{n}(m)$ are the magnitude and phase of the range-compressed data for the $n^{\text {th }}$ range bin respectively. The uncompensated phase error $\phi_{e}(m)$ along the synthetic aperture is the same for all range bins of interest and independent of $n$ [6]. The main four processing steps in PGA algorithm are explained in more details in the rest of this section.

\section{A. Circular shifting:}

First, those range bins need to be selected, that contain the clearest information about the phase errors [7]. Different approaches have been employed for this purpose. The approach applied here is to select range bins with the largest amount of energy. This assumes that the phase of strong reflecting objects is measured the most accurately and consequently results in the best estimate for the phase errors. For all selected range bins the azimuth cell with the strongest response is circularly shifted to image center. This means that cells that would be shifted off the edge of the array are instead wrapped around and shifted in from the opposite edge. This shifting removes the frequency offset in the corresponding Fourier domain due to the azimuth position of the scatterer, and in doing so, aligns point scatterers, subsequently improving the signal-to-noise ratio for the phase estimation. Fig.2(b) shows a circular shifted image for the image shown in Fig.2(a).

\section{B. Windowing:}

The next step is windowing [7]. The effect of windowing is to preserve the width of the dominant blur while removing data that do not contribute to phase error estimation. The input data then have the highest signal-to-noise ratio. The major problem for windowing is the selection of the window size. When the window is chosen too large, multiple images can become present from one scatterer. This is due to effects that are taken into account and corrected for but which are not due to movement of the platform, for example interference effects from another target when the window is chosen too small, not all of the motion error effect is taken into account, and focusing will no longer be sufficient. Because PGA is an iterative process, the window size needed will decrease after each iteration, as the error is reduced, a progressively decreasing window technique is used here to determine window size. Fig.2(C) shows a windowed image for the circular shifted image shown in Fig.2(b).

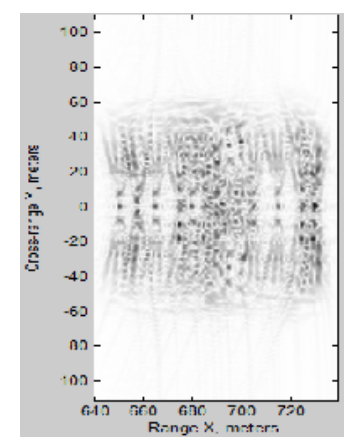

(a)

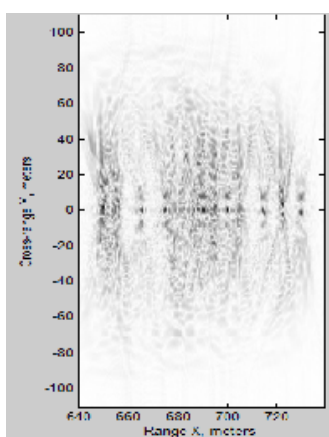

(b)

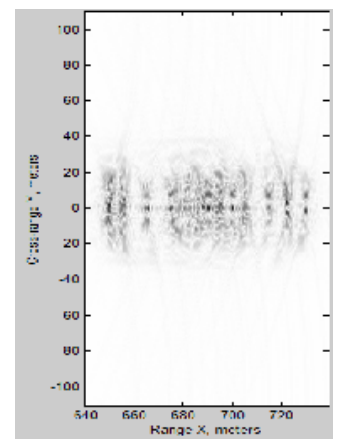

(c)

Fig. (2) shows a simulated SAR image with 23 scattereres; (a) blurred image,

(b) circular shifted image, (c) circular shifted and windowed image. 


\section{Phase gradient estimation}

After the image data is circularly shifted and windowed, the phase gradient is estimated. The rangecompressed phase history data $\mathrm{G}_{\mathrm{n}}(m)$ that can be obtained by inverse Fourier transform the shifted and windowed image data $\mathrm{g}_{\mathrm{n}}(m)$ can be written as:

$$
\mathrm{G}_{\mathrm{n}}(m)=\left|\mathrm{G}_{\mathrm{n}}(m)\right| \exp \mathrm{j}\left[\phi_{e}(m)+\phi_{n}(m)\right]
$$

Here $\phi_{n}(m)$ is the scatterer-dependent phase function for the $n^{\text {th }}$ range bin, $\phi_{e}(m)$ is the phase error function, which is range independent. The linear unbiased minimum variance (LUMV) estimate of the gradient of the phase error $\phi_{e}(m)$, is given by [3],

$$
\begin{aligned}
& \hat{\dot{\phi}}(m)=\frac{\sum_{n} \operatorname{Im}\left\{G_{n}^{*}(m) \mathcal{G}_{n}^{\&}(m)\right\}}{\sum_{n}\left|G_{n}(m)\right|^{2}} \\
& \hat{\phi}(m)=\phi_{e}^{\&}(m)+\frac{\sum_{n}\left|G_{n}(m)\right|^{2} \phi_{n}^{\&}(m)}{\sum_{n}\left|G_{n}(m)\right|^{2}}
\end{aligned}
$$

This LUMV estimate yields the gradient of the true phase error $\phi_{e}^{\&}(m)$, plus an error term that has been made as small as possible by the circular shifting and windowing operations. The PGA phase gradient estimation kernel in (4), is only one of a number of phase gradient estimation formulas.

\section{Iterative phase correction:}

The phase gradient is integrated and the phase is then applied to the original selected data in order to compensate the phase error [7]. All the described steps are then repeated. Thus, the altered phasehistory data set is Fourier transformed back to the image domain, circular shifted and windowed (with a new window size), and the phase error obtained. These steps are repeated until the rms error drops to a few tenths of a radian; the image is well focused and will not improve with additional iterations [2].

\section{Simulation Results:}

The simulated SAR images are generated by using a MATLAB programs for pulsed spotlight SAR simulation and reconstruction, this simulated SAR images of area size $(100 \mathrm{~m} \times 200 \mathrm{~m})$. Besides the visual comparison between the blurred and focused images, or between the input phase error function and the estimated one, the performances of autofocus algorithms are evaluated also by calculating the rms value of the residual phase error. Simulated SAR image are generated, it represents 23-point scatterers distributed in arbitrary manner as shown in Fig. 4(a). This image is corrupted by a multicycles sinusoidal phase error with amplitude $1.5 \pi \mathrm{rad}$ peak-to-peak, (3.3322 $\mathrm{rad} \mathrm{rms}$ ) as shown in Fig.3.

The blurred image is shown in Fig.4(b). PGA algorithm is used to focus this blurred image, Fig.5 shows the rms of the residual phase error versus the number of PGA iterations. It is quite clear that the PGA reaches a minimum $\mathrm{rms}$ after five iterations $(0.0027 \mathrm{rad})$, and its performance will not improve after more iterations. The residual phase error after five iterations is shown in Fig. 4(a), finally, Fig.4(c) 
shows the focused image obtained after five iterations of the PGA algorithm. This experiment show that, PGA algorithm requires five iterations to focus the image, typically, many literature show that PGA requires [4 6] iterations to focus the blurred image.

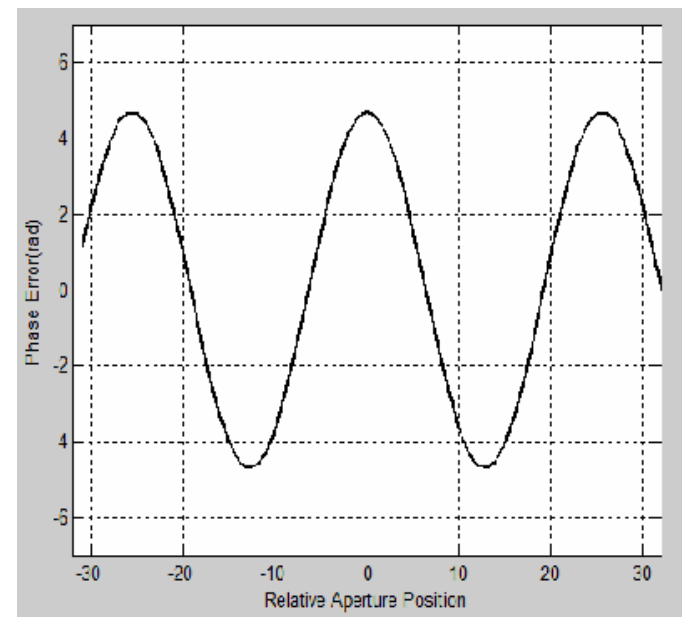

Fig. (3) Sinusoidal phase error function.

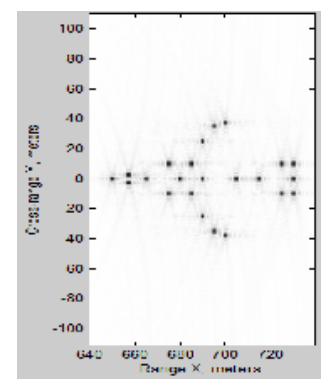

Fig. (4) (a) Simulated images for PGA performance test; (b) Image blurred with sinusoidal phase error;

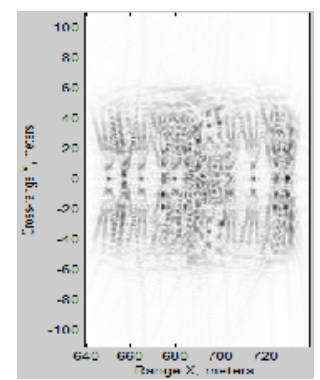

(b)

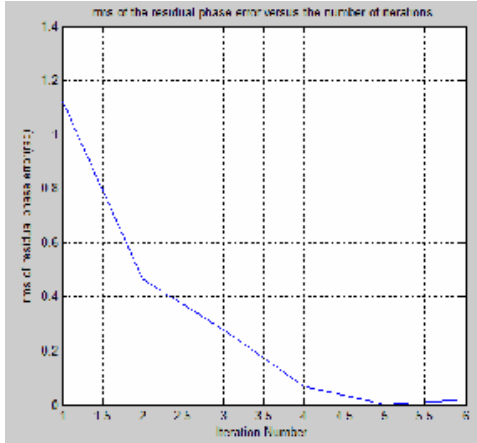

Fig. (5) The rms of residual phase error versus the iteration number for the simulated SAR image

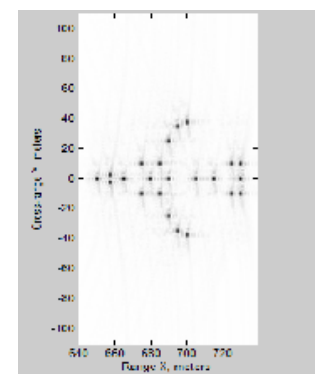

Fig. (4) (a) Simulated images for PGA performance test; (b) Image blurred with sinusoidal phase error; (c) PGA autofocus results after five iterations

\section{A suggested graphical user interface (GUI):}

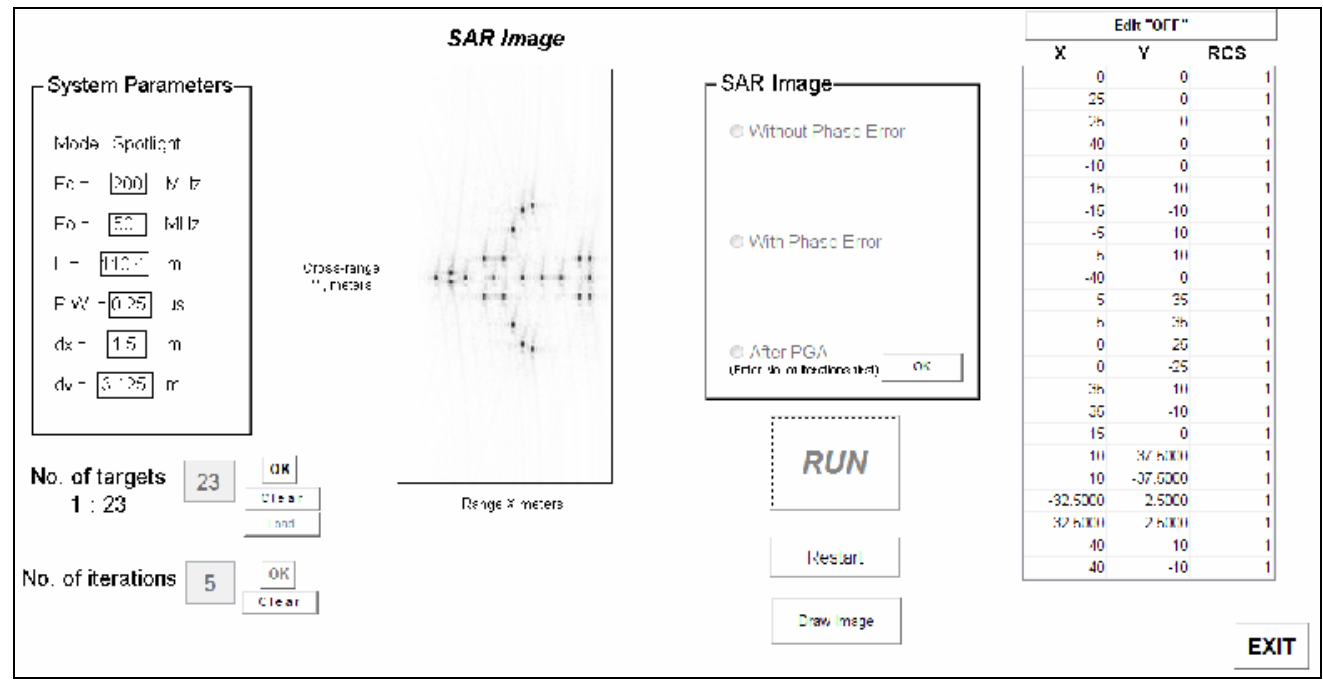

Fig. (6) Graphical User Interface (GUI) 
From the GUI, the user can enter, edit, and modify SAR and scene parameters, number of targets, number of iterations, and target position as shown in fig. 6. Desired SAR image can be viewed with/without phase error. The corrected output image is obtained by selecting "After PGA" button, which will appear at the middle of the GUI window.

\section{Conclusions:}

The PGA is a robust technique for SAR autofocus for different reasons, its nonparametric method (different forms of phase errors), has a good performance over a wide range of applications (independent on SAR scene), does not need for a strong isolated scatterer in image, works well on both high and low signal-to-clutter ratio images, and no human intervention.

\section{References:}

[1] M. P. G. Otten, "Comparison of SAR autofocus algorithms," in Proc. Military Microw., 1990, pp. 362-367.

[2] Wahl, D.E., P.h. Eichel,D.C. Ghiglia, and C.V. Jakowatz, Jr., "Phase Gradient Autofocus- A Robust Tool for High Resolution SAR Phase Correction", IEEE Trans. Aerospace Electron. Syst., VOL. 30, No. 3, pp 827-834, July 1994.

[3] Carrara W.G.,Goodman R.S., and Majewski R.M.,"Spotlight Synthetic Aperture Radar." Artech House, Boston, London, 1994.

[4] Oliver, C.J, "Understanding Synthetic Aperture Radar Images", Artec House, INC,1998.

[5] M.Kayton, and W.R. Fried, "Avionics Navigation Systems" John Wiely \& Sons, Inc 1997.

[6] Eichel, P.H, et al. "Phase Correction System for Automatic Focusing of Synthetic Aperture Radar", United States Patent No. 4,924,229, May 8, 1990.

[7] W. L. Van Rossum, M. P. G. Otten, R. J. P. Van Bree "Extended PGA for Range Migration Algorithms" IEEE Trans. Aerospace Electron. Syst., Vol.42, No.2 April 2006. 